\title{
Liver X Receptor-Like Family
}

National Cancer Institute

\section{Source}

National Cancer Institute. LiverX Receptor-Like Family. NCI Thesaurus. Code C128275.

A nuclear receptor family that binds oxysterols or bile acids and, after forming a heterodimer with a member of the retinoid X receptor (RXR) family, modulates the expression of genes that are involved in cholesterol, fatty acid, and glucose homeostasis. The members of this family include oxysterols receptor LXR-alpha (NR1H3), oxysterols receptor LXR-beta (NR1H2) and bile acid receptor (NR1H4). 